\title{
Mild encephalopathy with reversible splenial lesion associated with echovirus 6 infection: a case report and review of the literature
}

\author{
Enrico Masiello $^{1 \odot}$, Antonio Gatto ${ }^{1 \odot}$, Ilaria Lazzareschi ${ }^{1,2}{ }^{\circ}$, Donato Rigante ${ }^{1,2}$, \\ Paolo Mariotti ${ }^{3 \odot}$, Piero Valentini ${ }^{1,2 \odot}$ \\ ${ }^{1}$ Institute of Pediatrics, Fondazione Policlinico Universitario A. Gemelli IRCCS; ${ }^{2}$ Università Cattolica Sacro Cuore; ${ }^{3}$ Department of \\ Pediatric Neurology, Fondazione Policlinico Universitario A. Gemelli IRCCS, Rome, Italy.
}

\begin{abstract}
Background. Mild encephalopathy with reversible splenial lesion (MERS), a clinic-radiological syndrome distinguished by reversible encephalopathy onset, has been increasingly recognized in Caucasian children.

Case. We describe a MERS case in a previously healthy 4-year-old girl admitted to the hospital with abnormal consciousness level, muscle weakness, dysphagia and dysarthria after a 4-day history of diarrhea and fever. Magnetic resonance imaging (MRI) of the brain showed hyperintensity in the corpus callosum splenium. Electroencephalogram was normal and cerebrospinal fluid (CSF) culture negative. The stool sample was positive for Echovirus 6 and serology test confirmed the infection. The child's condition gradually improved and the MRI, after 15 days, depicted a normal brain. Only a mild speech impairment was persistent at discharge, which disappeared one month later. We performed a literature review about pediatric MERS cases demonstrating that infectious agents have been rarely isolated in CSF.
\end{abstract}

Conclusion. MERS has an overall good prognosis independently from the treatment approach; this is confirmed by our case - one of the first reported with an Echovirus 6-related encephalopathy.

Key words: mild encephalopathy with reversible splenial lesion (MERS), echovirus, encephalopathy, child.

Mild encephalopathy with reversible splenial lesion (MERS) is a disorder characterized by prodromal symptoms such as fever, cough, vomiting or diarrhea, followed by mild encephalopathy 1-7 days later with a documented reversible splenial lesion. Lesions are typically hyperintense in T2-weighted brain magnetic resonance imaging (MRI) and demonstrate a transiently homogeneous low apparent diffusion coefficient (ADC). Takanashi et al. ${ }^{1}$ proposed to classify this encephalopathy in MERS type I, describing patients with an isolated splenium corpus callosum (SCC) lesion and in MERS type II for patients with extensive

$\bowtie$ Antonio Gatto

antonio.gatto@policlinicogemelli.it

Received 9th April 2019, revised 15th June 2019, accepted 3rd July 2019. white matter and/or lesions involving the entire corpus callosum (CC). The most relevant and common neurological MERS symptoms, which tend to completely recover within 1 month, are behavioral changes, consciousness disturbance and seizures. ${ }^{2-5}$

Numerous infectious diseases are associated with MERS in children: rotavirus (RV), ${ }^{6-14}$ cytomegalovirus (CMV), ${ }^{15-17}$ influenza $\mathrm{A}$ and $\mathrm{B}^{5,16-28}$ parainfluenza, ${ }^{29}$ mumps ${ }^{2-30,31}$ as well as other viral agents such as adenovirus, ${ }^{16}$ human herpesvirus-6 (HHV-6), ${ }^{32}$ human herpesvirus-1, ${ }^{17}$ parvovirus B19, ${ }^{33}$ enterovirus $(\mathrm{EV})^{17}$ and Epstein-Barr virus (EBV). ${ }^{34}$ Also bacterial infections have been described related to MERS cases, namely Escherichia coli, ${ }^{28}$ Enterococcus faecalis, ${ }^{35}$ Salmonella, ${ }^{16}$ Campylobacter jejuni ${ }^{28}$ and Mycoplasma pneumoniae. .7,28,36-44 $^{2}$ 
Herein we describe a pediatric patient with Echovirus 6 infection and transient isolated SCC lesion on the brain MRI followed by extensive medical literature review about MERS in children.

\section{Case Report}

A previously healthy 4-year-old Caucasian girl with a 4-day-history of fever and diarrhea and mildl dehydration, was referred to our hospital in summer 2018. Patient's and family medical history was unremarkable. Physical examination on admission showed a body temperature of $38^{\circ} \mathrm{C}$ with a heart rate of 130 beats per minute, respiratory rate of 36 breaths per minute, oxygen saturation of $98 \%$ breathing room air and blood pressure of 103/64 $\mathrm{mmHg}$. Chest and abdominal examination was normal. Neurological examination revealed an abnormal consciousness level, muscle weakness, dysphagia and dysarthria. The patient was responsive to painful stimuli with Glasgow Coma Scale score of 12 (eyes 3, verbal 4, motor 5), showed normal reflexes in the extremities; Kernig's and Babinski's signs were absent. Peripheral blood analysis showed a white blood cell count of $6970 / \mu \mathrm{L}$, hemoglobin $10.6 \mathrm{~g} / \mathrm{dl}$, platelet count 325.000/ $\mu \mathrm{L}$ and C-reactive protein $130 \mathrm{mg} / \mathrm{L}$ (normal value: $<5)$. Biochemical parameters and blood gas analysis were normal. Lumbar puncture was performed, and cerebrospinal fluid (CSF) analysis revealed 125 white cell $/ \mathrm{ml}$, increased protein $50 \mathrm{mg} / \mathrm{dl}$ and normal glucose $52 \mathrm{mg} /$ dl. Isotonic fluids and empirical intravenous antibiotic treatment with ceftriaxone and intravenous acyclovir were started. Real TimePolymerase Chain Reaction (RT-PCR) to detect $\mathrm{CMV}, \mathrm{EBV}, \mathrm{EV}$, influenza viruses and herpes viruses in CSF were negative. Bacterial CSF cultures were also negative. Specific serum IgM antibodies (ELISA) against EBV, influenza virus, parainfluenza viruses, parvovirus B19, herpes viruses and $\mathrm{CMV}$ were negative. Conversely, serological tests for influenza A, adenovirus, HHV-6 and varicella demonstrated previous infections. RV detection test was negative. RT-
PCR was also negative for influenza A and B in the pharyngeal swab. Urine analysis was normal. Electroencephalogram (EEG) revealed high-voltage slow waves on both temporal regions, with no paroxysmal discharge activity. Brain MRI, on day 2 of hospitalization, showed a focal high-signal lesion of SCC on T2-weighted, fluid-attenuated inversion recovery (FLAIR) and diffusion-weighted images (Fig. 1). Specific serum IgM and IgA antibodies (ELISA) against Echovirus were positive and the stool culture confirmed an acute infection with Echovirus 6. We interrupted ceftriaxone and acyclovir therapy on day 5. In consideration of persistent neurological symptoms such as muscle weakness, dysphagia and dysarthria combined with drowsiness and ataxia, brain MRI results, we initiated intravenous methylprednisolone treatment (2 $\mathrm{mg} / \mathrm{kg} /$ day) for 5 days and continued the treatment with prednisone per os $(2 \mathrm{mg} / \mathrm{kg} /$ day) for 2 weeks and subsequent decalage. A progressive clinical improvement was already noted on day 7 of hospitalization and continued until neurological symptoms disappeared, when the girl turned to a normal consciousness level and restarted drinking fluids. Fifteen days after admission, follow-up brain MRI and EEG were normal. The patient was discharged without neurological sequelae, except for a residual speech impairment, which had disappeared at her one-month followup visit. Informed consent was obtained by the parents for all procedures and for the publication of this case.

\section{Discussion}

EV epidemic outbreaks occur in summer or early fall in temperate regions. Echovirus 6 is one of the most frequently detected EV worldwide. ${ }^{45}$ EVs are classified in 4 species: (a) human enterovirus $\mathrm{A}$ (coxsackie virus A2-A8, A10, A12, A14, and A16; EV71, 76, 89, 90, and 91), (b) human enterovirus $B$ (coxsackie virus A9 and CVB1-CVB6; echovirus 1-7, 9, 11-27, and 29-33; EV69, 73-75, 77-88, 97, 100, and 101), (c) human enterovirus $C$ (coxsackie virus A1, A11, A13, A17, A19-A22, and A24; polioviruses 1-3; 

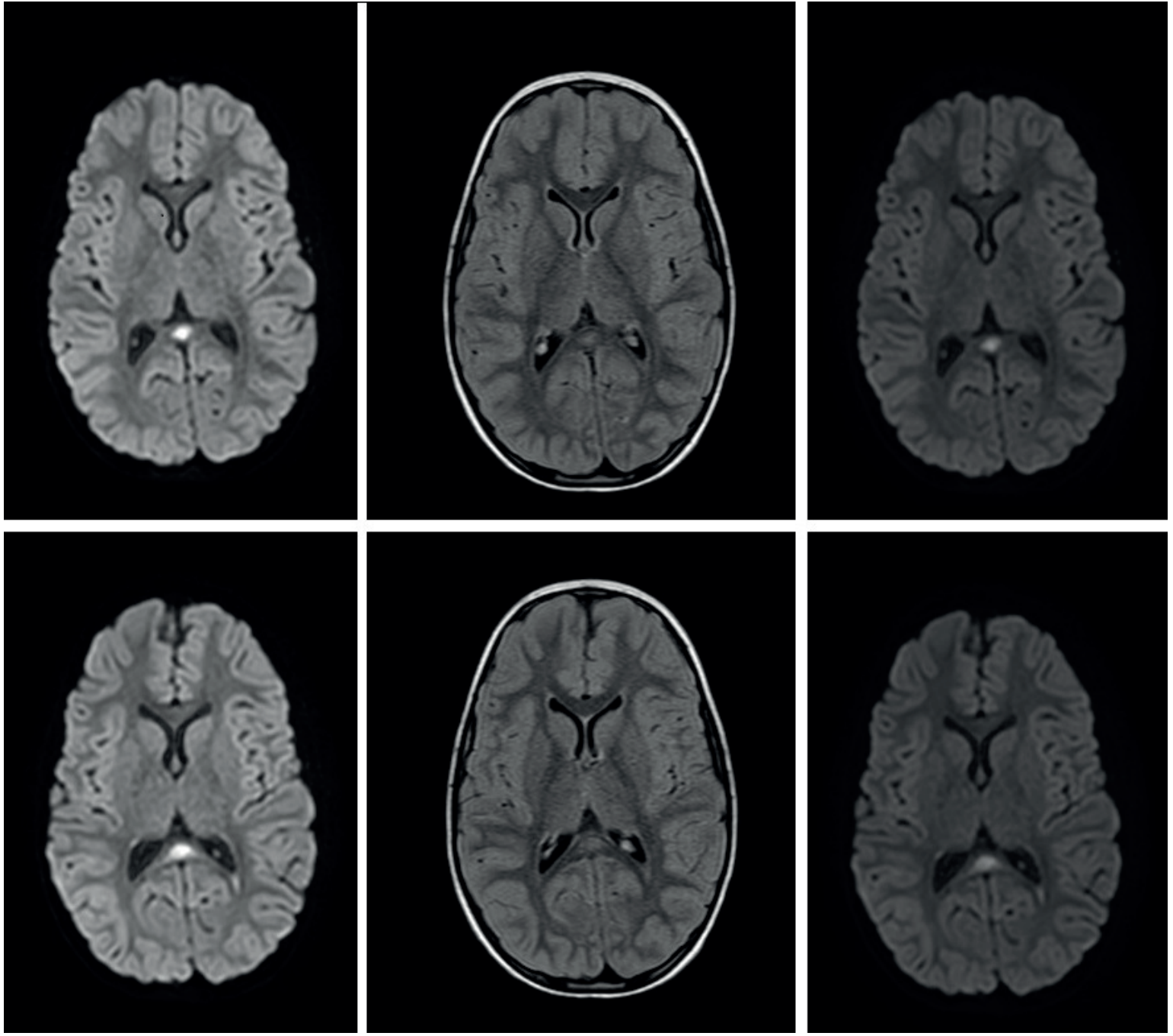

Fig. 1. MRI imagines on day 2 with hyperintensity in the SCC.

EV96), and (d) human enterovirus D (EV68 and EV70). ${ }^{46}$ Echovirus (E) is the major causative agent of aseptic meningitis, and E6, E9, E11, E13, E19, E30 are the most common EV types detected in patients with aseptic meningitis. Consciousness disturbance, ataxia, acute muscle weakness, dysarthria and dysphagia, as reported in our patient, are typical neurological symptoms of EV-related encephalitis. Skin rashes and diarrhea are common nonneurological symptoms in EV infections. ${ }^{45}$

Brain MRI, in the presented case, revealed a transient splenial lesion, based on which we diagnosed MERS, a benign disorder characterized by homogenously reduced diffusion lesions (type I MERS), occasionally associated with extensive white matter and/or entire callosal lesions (type II MERS) on brain MRI. ${ }^{1,2,47}$ Kobata et al. ${ }^{6}$ eported for the first time a RV-related MERS case. In 2004, Tada et al. ${ }^{2}$ described MERS as a new encephalopathy with a mild clinical course and good outcome.

We performed a literature review on pediatric MERS cases until July 2018 using Pubmed and Google database for English, Italian and Japanese language publications to clarify clinical features, etiology, neuroimaging and prognosis of this condition. The search was performed using the following keywords: "enterovirus", "echovirus", "encephalitis", "encephalopathy", 
"mild encephalitis/encephalopathy with a reversible splenial lesion", "MERS" and "pediatric MERS". We identified 165 cases, including 52 English-language full reports (145 cases), 1 Japanese-language full report (1 case $^{48}$ and 9 English-language abstracts (11 cases). Inclusion criteria were age less than 18 years and encephalopathy by means of brain MRI showing reversible hyperintensity of the splenium of the corpus callosum.

In the 165 pediatric MERS cases, mean age at time of diagnosis was 5.04 years (range 1 day18 years) and male/female ratio (88/77) was 1.14. Clinical characteristics, laboratory data, neuroimaging results, and patient outcomes are summarized in Table I.

Considering the demographic distribution of patients, MERS occurs mostly in South East Asia, with 84 Japanese cases, 53 Chinese, 9 Australian and 9 Turkish cases, 3 Belgium cases, 1 case from Switzerland, United Kingdom, Poland, Malaysia, Korea, USA and Italy, respectively. Some Australian reports documented the syndrome predominantly in the Caucasian population, indicating an epidemiologic correlation to local virus circulation instead of genetic association. ${ }^{16,19}$ Clinical surveillance in Europe demonstrated variable non-polio infection distribution across the years. An increased occurrence rate of $60 \%$ related to Echovirus 6 infection with neurological symptoms in patients younger than 7 years was documented in Netherlands from January until August 2016. ${ }^{49}$

Consciousness disturbance (CD; GCS $<13$ ) was the most common (93/165, 56.3\%) neurological symptom in MERS patients, followed by seizures $(77 / 165,46.6 \%)$ and delirious behavior (DB) $(55 / 165,33.3 \%)$. As described by Kashiwagi et al. ${ }^{5}$, DB symptoms are divided as follows: visual hallucination, nonvisual sensory misperceptions, emotional changes (such as laughter and fear), incoherent speech, purposeless movements, and impulsive behavior. Among the 55 cases with $\mathrm{DB}$, clear delirium occurred in 13/55 (23.6\%), irritability in $13 / 55(23.6 \%)$, hallucinations in 5/55 $(9.1 \%)$, purposeless movements in $4 / 55(7.3 \%)$ and abnormal behavior in 3/55 (5.4\%). Movement disorders were described in 16/165 patients $(9.7 \%)$, mainly ataxia in $12 / 16(75.0 \%)$, tremor in $3 / 16(18.7 \%)$ and gait disturbance in $1 / 16(6.2 \%)$. Speech impairment was reported in $16 / 165$ patients (9.7\%), dysarthria in 5/16 (31.2\%), slurred speech in 5/16 (31.2\%), abnormal speech in $5 / 16(31.2 \%)$, and mutism in $2 / 16(12.5 \%)$. Motor deficits were described in 11/165 (6.6\%) patients, $5 / 11(45.4 \%)$ showed signs of motor deterioration, $2 / 11$ (18.2\%) muscle weakness, $1 / 11(9.1 \%)$ upper arm paresis, $1 / 11(9.1 \%)$ lower arm sensorimotor polyneuropathy, $1 / 11$ (9.1\%) dominant hemiplegia and 1/11 (9.1\%) akinetic mutism. Cranial nerve deficits such as eye movement disorders, ophthalmoplegia and strabismus were reported in 4/165 patients $(2.4 \%)$, blindness in $3 / 165(1.8 \%)$, dizziness in $2 / 165(1.2 \%)$, facial palsy in $1 / 165(0.6 \%)$ and pseudobulbar palsy in 1/165 $(0.6 \%)$.

Fever $(117 / 165,70.9 \%)$ was the most common non-neurological prodromal symptom, followed by gastrointestinal symptoms such as abdominal pain, vomiting and diarrhea (77/165, $46.6 \%)$, cough $(29 / 165,17.6 \%)$ and headache $(21 / 165,12.7 \%)$. Only one patient had clinical signs of sepsis. ${ }^{50}$

MERS-associated infectious agents were identified in 106/165 patients (64.2\%): RV in 26/165 (15.7\%), Mycoplasma pneumoniae in 19/165 $(11.5 \%)$, influenza $\mathrm{A}$ in $14 / 165$ (8.5\%), mumps virus in $8 / 165(4.8 \%)$, adenovirus in $6 / 165$ (3.6\%), influenza B in 6/165 (3.6\%), CMV in $3 / 165(1.8 \%)$, Enterococcus faecalis in 3/165 (1.8\%), EBV in 3/165 (1.8\%) - one had also an EBVrelated hemophagocytic lymphohistiocytosis - herpesvirus in 2/165 (1.2\%), coxsackie virus in 2/165 (1.2\%), Echovirus in 2/165 (1.2\%), Escherichia coli in 2/165 (1.2\%), parainfluenza virus, Salmonella gastroenteritis, $\mathrm{HHV}$ 6 infection, Campylobacter jejuni, Klebsiella pneumoniae, parvovirus B19, respiratory syncytial virus, dengue virus, Streptococcus pneumoniae and group B Streptococcus in only 1 patient $(0.6 \%)$, respectively. MERS was 


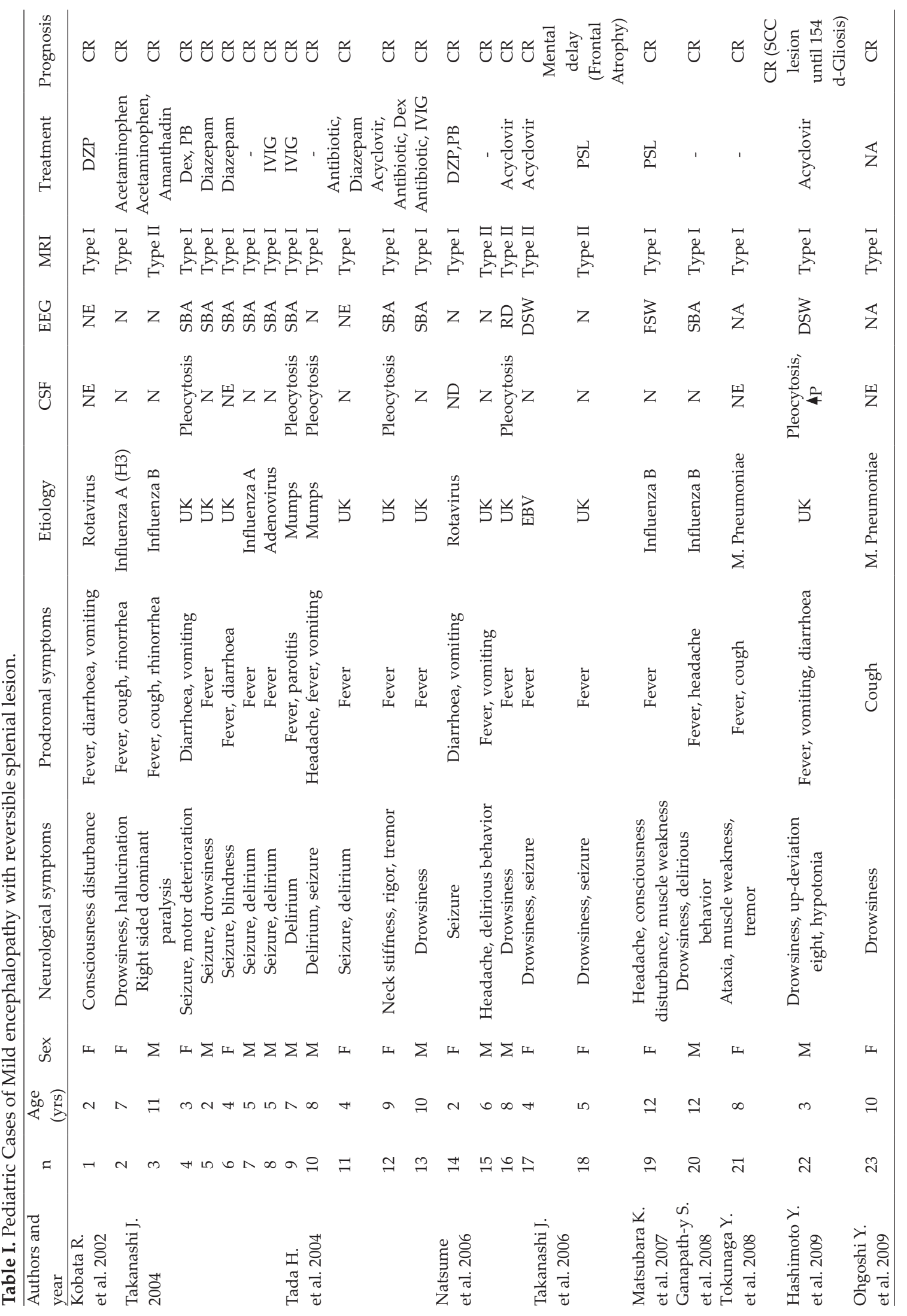




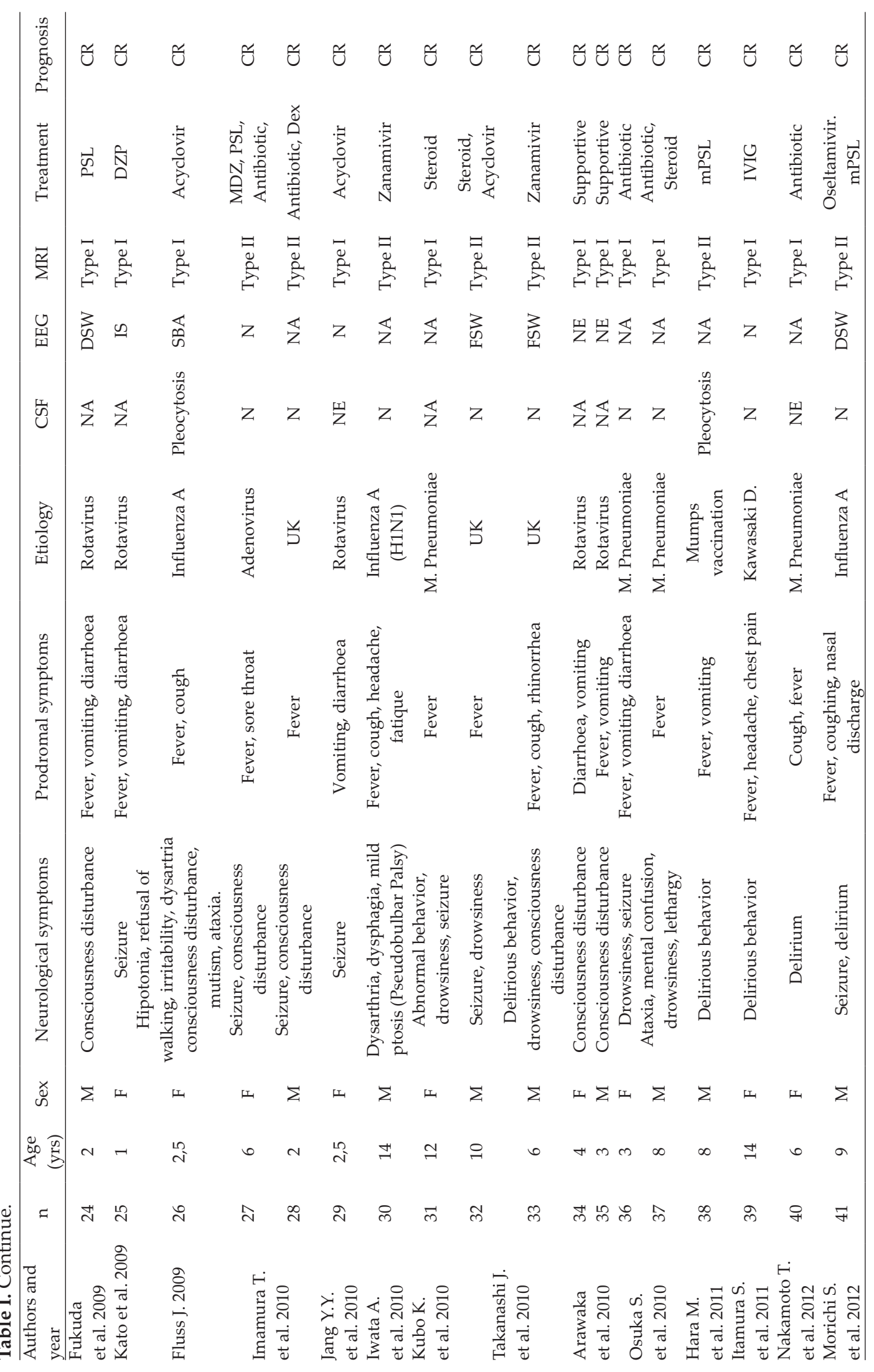




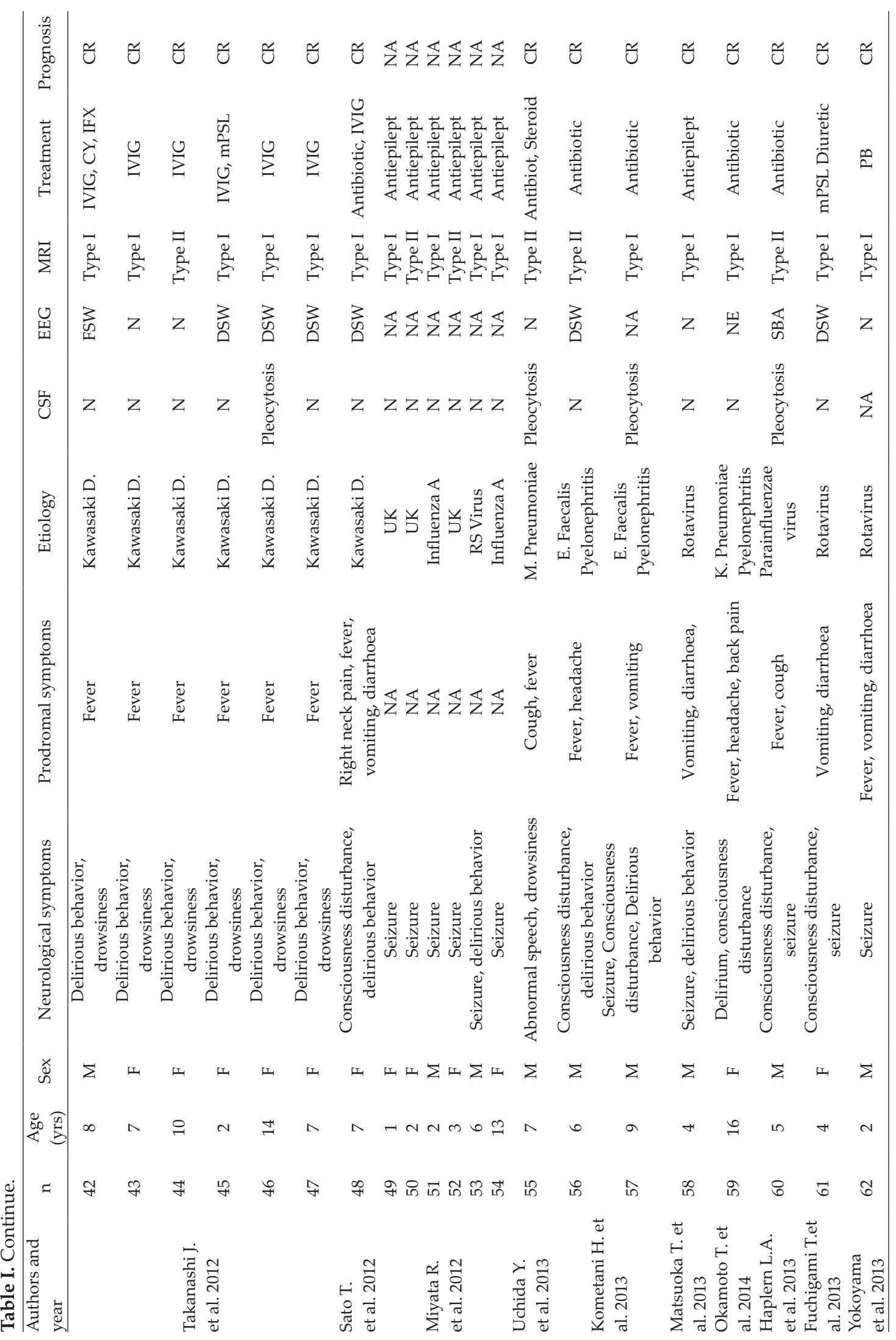




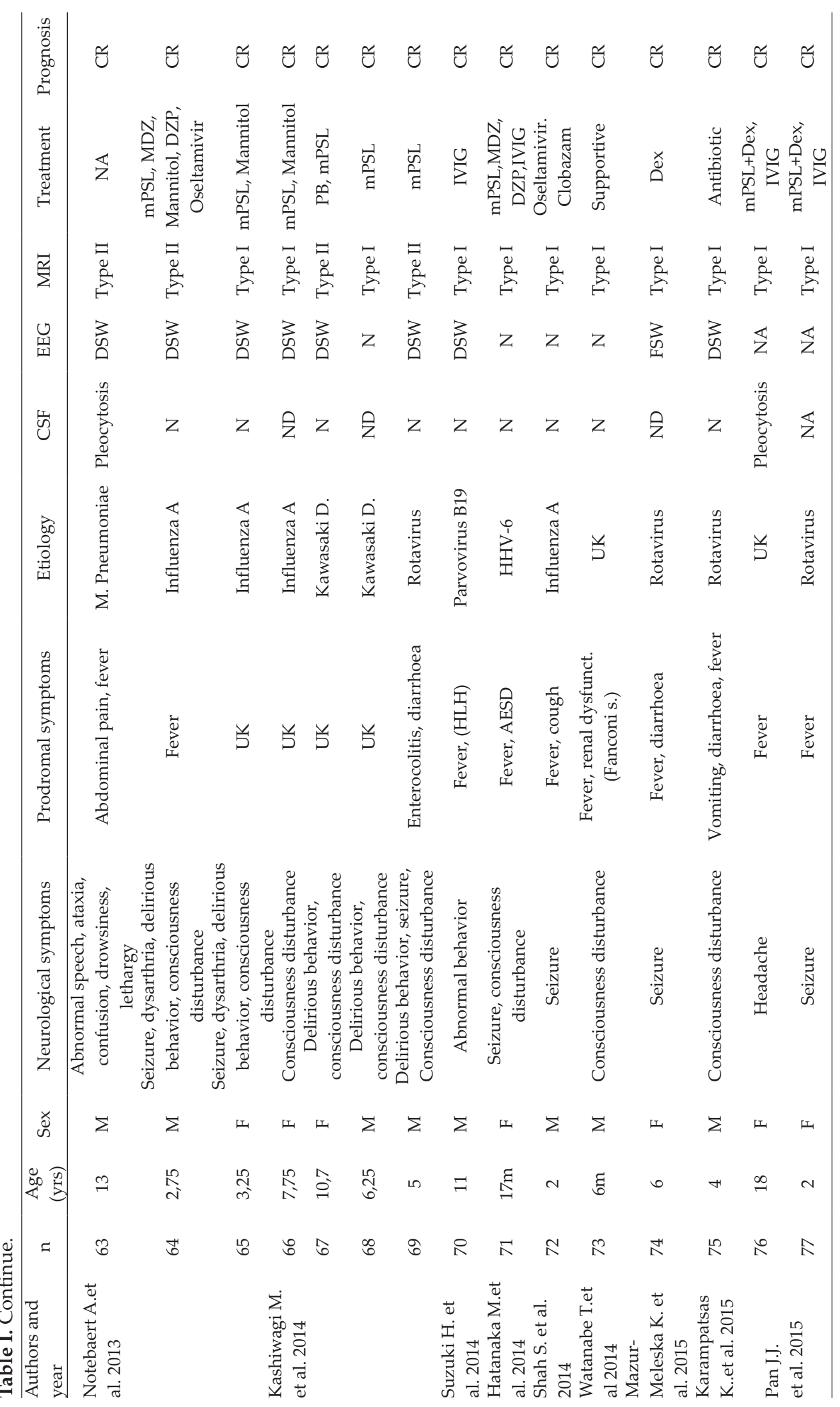




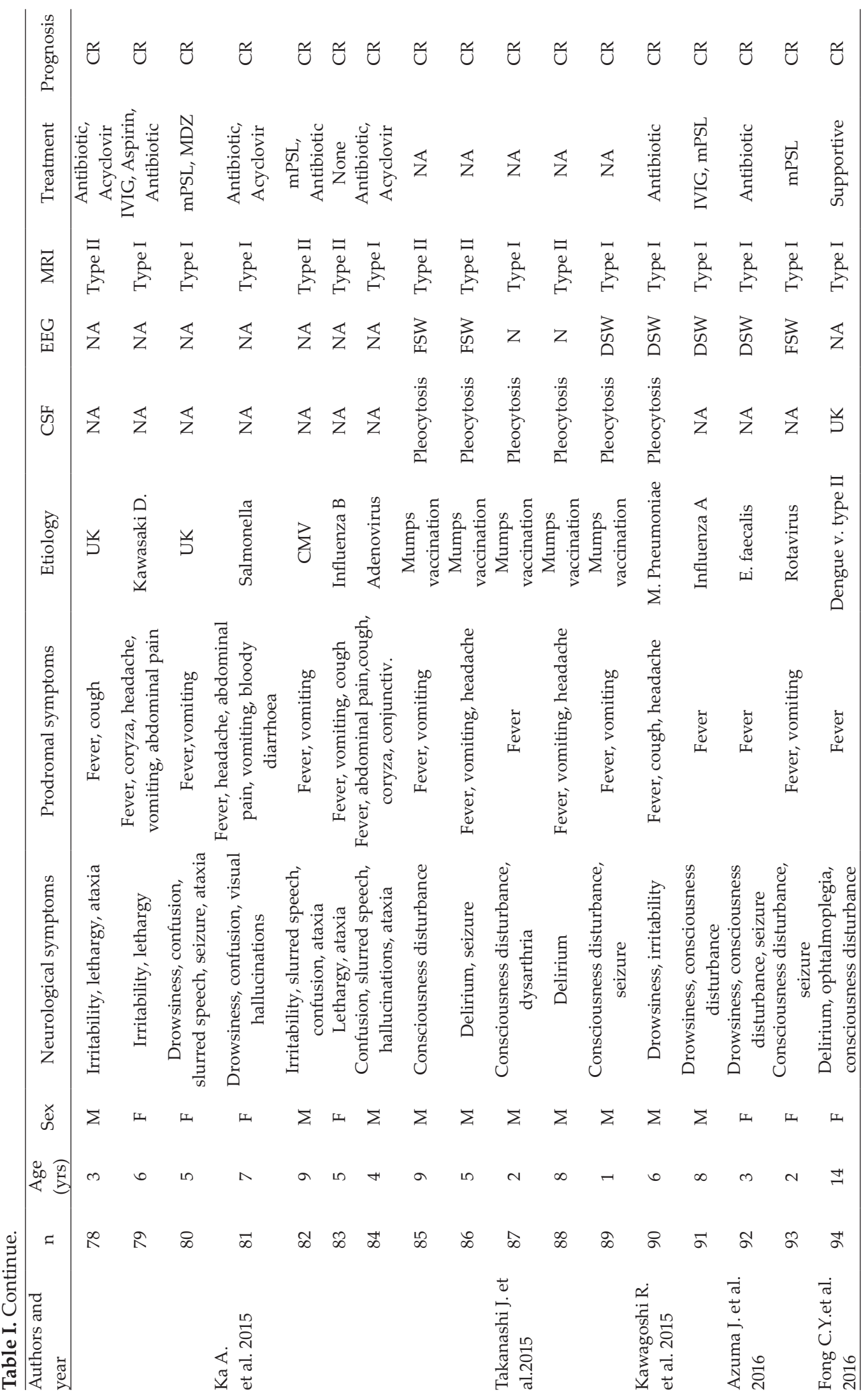




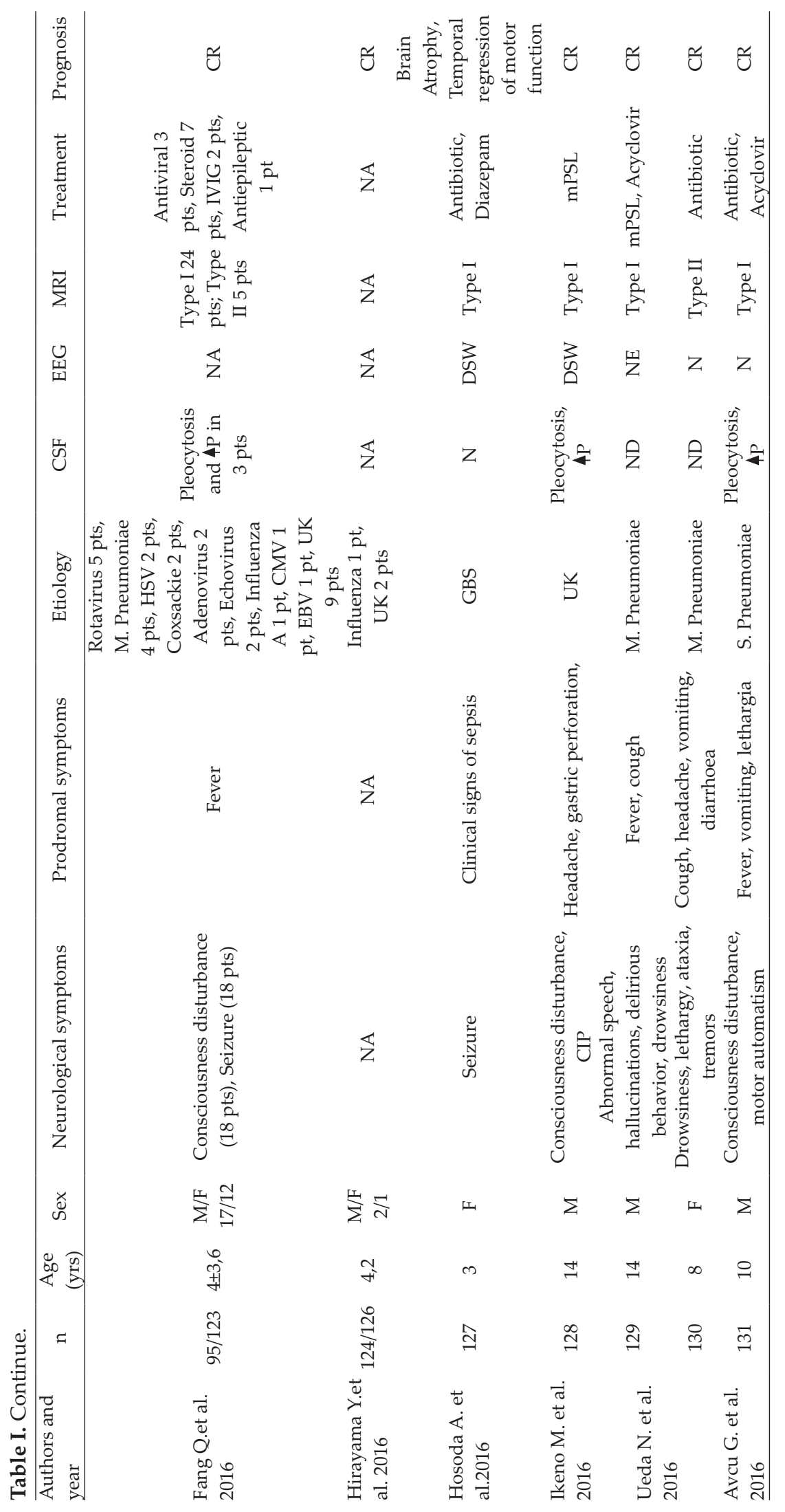




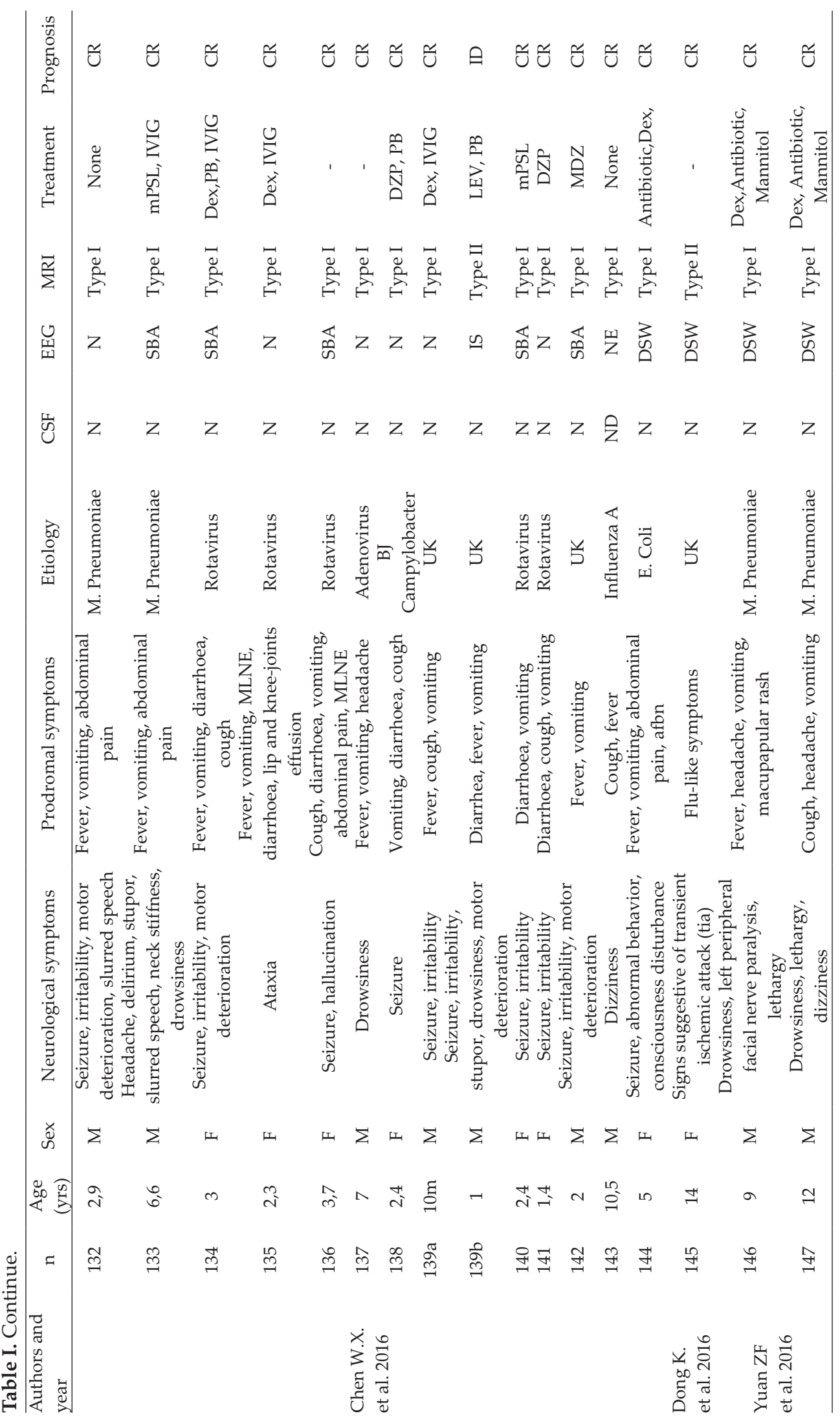




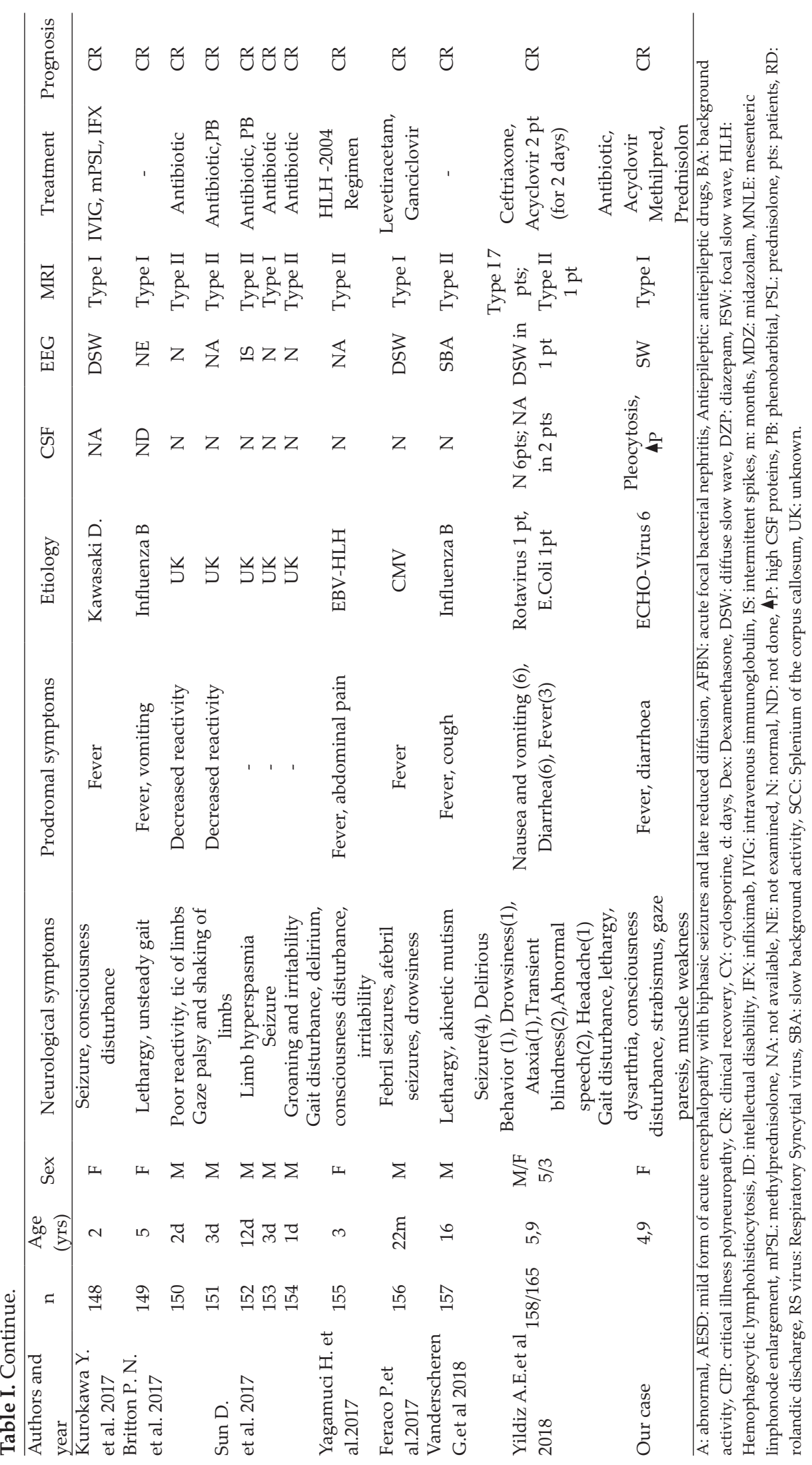


associated with Kawasaki disease in 12/165 $(7.3 \%)$ patients. Microbiologic and serologic examinations were negative in 59/165 (35.7\%) patients, and no infectious pathogens were identified in these cases.

We analyzed the association between etiological agents and symptoms. CD symptoms 93/165 $(56.4 \%)$ were most frequently related to Mycoplasma pneumoniae in 12/93 (12.9\%) patients, $\mathrm{RV}$ in $8 / 93(8.6 \%)$ and influenza virus in $6 / 93(6.4 \%)$. About patients developing seizures $(77 / 165,46.7 \%)$, RV infection was identified in $14 / 77(18.2 \%)$ and influenza in 6/77 (7.8\%). Considering cases with DB symptoms (55/165, $33.3 \%$ ) the most frequent etiological agents were influenza A virus in $7 / 55$ patients $(12.7 \%)$, RV in 6/55 (10.9\%) Mycoplasma pneumoniae in 6/55 $(10.9 \%)$ and mumps virus in 5/55 (9.0\%). In 10/55 of patients with DB (18.2\%) Kawasaki disease was confirmed. Movement disorders described in 16/165 cases (9.7\%) occurred most frequently in patients with Mycoplasma pneumoniae infections $(4 / 16,25.0 \%)$, and all these cases had ataxia. Out of 16/165 cases characterized by speech impairment $(9.7 \%)$, the most frequent pathogens identified were Mycoplasma pneumoniae in 5/16 (31.2\%), influenza A virus in 4/16 (25.0\%), influenza B in 1/16 (6.2\%), CMV in $1 / 16(6.2 \%)$, adenovirus in $1 / 16(6.2 \%)$, and mumps virus in $1 / 16(6.2 \%)$. In $4 / 5$ cases $(80.0 \%)$ with dysarthria an association with influenza A virus was demonstrated. Mutism was reported in 2 patients with influenza virus (A in 1 case and $\mathrm{B}$ in the other one).

Lumbar puncture was performed in 135/165 $(81.8 \%)$ patients, and CSF pleocytosis $(>10$ WBCs/ $\mu \mathrm{l})$ was diagnosed in 24/165 (14.5\%) patients, $7 / 24 \quad(29.2 \%)$ showed increased protein levels. 17/24 (70.8\%) CSF pleocytosis patients were described as MERS type I and $7 / 24(29.2 \%)$ patients as type II. No correlation between pleocytosis and worse outcome could be observed. CSF pleocytosis patietns had the following infections: mumps virus in $8 / 24$ (33.3\%), Mycoplasma pneumoniae in $3 / 24$ $(12.5 \%)$, Enterococcus faecalis in $1 / 24(4.2 \%)$, Streptococcus pneumoniae in $1 / 24(4.2 \%)$ and human parainfluenza virus type 3 in $1 / 24$ (4.2\%). In 2 patients, persistent pleocytosis were described for a period of more than 5 months. ${ }^{51-52}$ Identification of the infectious agent by RT-PCR on CSF specimens was performed in only 6 patients with mumps-related MERS, ${ }^{31-32}$ while in other patients diagnosis was achieved with a blood serological test or RT-PCR with throat swab. CSF glucose was normal in all cases.

Considering EEG, in 60/165 patients (36.4\%) EEG findings were abnormal: focal slow waves were described in 8/60 (13.3\%) (occipital waves in 5, parieto-occipital, temporo-occipital and frontal waves in 1, respectively); EEG revealed diffuse slow waves in 31/60 (51.7\%) (authors described high voltage slow wave in 7 cases), slow background activity in $17 / 60$ $(28.3 \%)$, intermittent spikes in 3/60 (5.0\%), and rolandic discharge in 1/60 (1.7\%). Analyzing the association between EEG results and etiological agents, influenza virus (both A and B) was observed in $8 / 60(13.3 \%), \mathrm{RV}$ in $7 / 60(11.7 \%)$, mumps virus in 4/60 (6.7\%), adenovirus, EBV, CMV, Escherichia coli and Enterococcus faecalis in $1 / 60(1.7 \%)$, respectively. Analyzing the association between EEG results and clinical syndrome diagnosis of Kawasaki disease was found in $6 / 60$ patients $(10.0 \%)$ with EEG abnormalities. Between patients with abnormal EEG findings, 38/60 (63.3\%) had CD symptoms, 26/60 (43.3\%) seizures and 19/60 (31.7\%) DB. In all patients, follow-up EEG was normal, as also in our case. Clinical follow-up in these 60 patients revealed a good prognosis, except for one MERS type II case reported by Chen et al. ${ }^{28}$, who developed intellectual disability.

Considering neuroimaging, 121/165 (73.3\%) were diagnosed as MERS type I. Diagnosis of MERS type II was reported in 44/165 (26.7\%) with hyperintensity lesions of CC in association with hyperintensity in the semioval center and parietal white matter bilaterally, or associated with diffuse hyperintensity of white matter (6 patients), or with hyperintensity in the center semiovale and periventricular symmetric hyperintense lesions (8 cases). In $3(2.0 \%)$ cases, authors did not specify the extension 
of hyperintensity lesions in the CC. Fluss et al. ${ }^{21}$ described a MERS type II case with an additional restricted diffusion area of the right dentate nucleus suffering clinical mutism. In 2006, Takanashi et al. ${ }^{3}$ reported a MERS type II with hyperintense asymmetric lesion in the gray matter of the prefrontal cortex with subsequent frontal atrophy and intellectual disability. Hyperintensity lesions in the SCC and in other brain areas on neuroimaging disappeared within 30 days in all cases, except in 1 patient with MERS type I, in whom the lesion could be still detected on brain MRI follow-up in T2weighted imagines after 134 day. CSF analysis showed pleocytosis and increased proteins. ${ }^{51}$

Data about treatments revealed that 35/165 patients $(21.2 \%)$ received antibiotic therapy after MERS onset, and 21/165 (12.7\%) antiviral treatment. Intravenous immunoglobulin was administered to $23 / 165$ (13.9\%) patients, in 9 cases in association with corticosteroids. Eleven patients received, as steroid therapy, only Dexamethazone; 23 patients, methilprednisolone (authors in 21 cases had specified a pulsed-dosed therapy; in 2 cases was prescribed also Dexamethazone) and 2 patients prednisolone. For 11 cases the corticosteroids treatment has not been specified. In 2 patients, infliximab was also administered (Table I).

The median recovery time was 13.3 days for MERS type I (though data about recovery time was only for $103 / 121$ (85.1\%) patients available) and 12.6 days for MERS type II (data about recovery time was for $34 / 44(77.3 \%)$ patients available).

Regarding prognosis, all patients completely recovered within 30 days, except for 4 patients. A long- term follow-up showed intellectual disability in 2 MERS type II cases. ${ }^{3,28}$ Notably, one of these patients had recurrent MERS episodes; the other patient showed brain lesions in regions usually not involved in MERS, as the prefrontal cortex and white matter. Another patient with MERS type I associated with recurrent Streptococcus group B sepsis had mild brain atrophy and motor function regression, which improved gradually in the long-term. ${ }^{50}$ Notebaert et al. ${ }^{44}$ described one Mycoplasma pneumoniae-related MERS type II case with residual ataxia and speech impairment, who fully recovered 4 months later. Fluss et al. ${ }^{21}$ described one case of influenza A-related MERS with a cerebellar lesion (in the right dentate nucleus) on MRI, presenting with dysarthria and ataxia; in this patient clinical recovery was obtained 1 month later.

Our literature review revealed two cases in which MERS and other encephalopathies overlapped. One case described, additional to MERS, febrile infection-related epilepsy syndrome (FIRES), characterized by intractable seizures and the other case acute encephalopathy with biphasic seizures and reduced diffusion (AESD), characterized by recurrent complex partial seizures. ${ }^{30,53}$

The exact relationship between radiological evidence and clinical condition of MERS patients is still unclear. In a retrospective study, Tada et al. $^{2}$ excluded any correlation between neuro-radiological features of brain lesions and neurological symptoms. Ueda et al. ${ }^{36}$ reported a longer clinical recovery time in MERS type II related to Mycoplasma pneumoniae infection than in type I, suggesting also a less benign course.

MERS pathogenesis is still unclear. Researchers hypnotized intramyelinic edema as a possible cause, which however cannot explain neonatal MERS occurrence considering the incomplete myelination in newborns. ${ }^{1,22,29,54,55}$ Kawasaki disease, an acute febrile systemic vasculitis, has been described in association with MERS, demonstrating a possible correlation to immune system abnormalities triggering the pathogenesis. ${ }^{53,56,57}$ Kometani et al. $^{58}$ reported a IL-6 elevation in the CSF of MERS patients associated with focal bacterial nephritis caused by Enterococcus faecalis. RT-PCR rarely detected infections in the CSF, suggesting an immunemediated mechanism in MERS pathogenesis. In our review, direct causative agents in CSF were identified only in 6 patients. ${ }^{31,32}$ Moreover, unknown genetic factors could 
be likely involved, and indeed recurrent and familial cases of MERS are described in medical literature. Neuroradiological features in familiar forms are also characterized by a more extensive brain involvement then in sporadic MERS cases. ${ }^{59,60}$

Recently, MERS has been increasingly recognized in Caucasian children, although none of cases had a recognized Echovirus 6 infection, as our patient. Infections (viral in $58 \%$ of cases), but also systemic inflammatory diseases (as Kawasaki disease) can be associated with MERS. Neurological manifestations occur shortly after prodromal symptoms and neuroimaging consents to substantiate MERS diagnosis. A short follow-up is necessary to confirm the transient nature of splenial lesions. The therapeutic approach in MERS may vary on a case-by-case basis; it remains unclear if a specific therapy might change the clinical history of the disease. Data of our review demonstrate that MERS is a disease with an overall good prognosis, as almost all patients reported a complete recovery of neurological symptoms within 30 days irrespective of treatment. Considering the low number of MERS cases and different severity degrees, multicentre studies are needed to clearly elucidate its pathogenesis and define treatment guidelines.

\section{REFERENCES}

1. Takanashi JI, Imamura A, Hayakawa F, Terada H. Differences in the time course of splenial and white matter lesions in clinically mild encephalitis/ encephalopathy with a reversible splenial lesion (MERS). J Neurol Sci 2010; 292: 24-27.

2. Tada H, Takanashi J, Barkovich AJ, et al. Clinically mild encephalitis/encephalopathy with a reversible splenial lesion. Neurology 2004; 63: 1854-1858.

3. Takanashi J, Barkovich AJ, Shiihara T, et al. Widening spectrum of a reversible splenial lesion with transiently reduced diffusion. AJNR Am J Neuroradiol 2006; 27: 836-838.

4. Takanashi JI. Two newly proposed encephalitis/ encephalopathy syndromes. Brain Dev 2009; 31: 521-528.
5. Kashiwagi M, Tanabe T, Shimakawa S, et al. Clinicoradiological spectrum of reversible splenial lesions in children. Brain Dev 2014; 36: 330-336.

6. Kobata R, Tsukahara H, Nakai A, et al. Transient MR signal changes in the splenium of the corpus callosum in rotavirus encephalopathy: value of diffusion-weighted imaging. J Comput Assist Tomogr 2002; 26: 825-828.

7. Karampatsas K, Spyridou C, Morrison IR, Tong CYW, Prendergast AJ. Rotavirus associated mild encephalopathy with a reversible splenial lesion (MERS)- case report and review of the literature. BMC Infect Dis 2015; 15: 446.

8. Yokoyama T, Yamada S, Doichi N, Kato E. Rotavirus-infected children with clinically mild encephalopathy with a reversible splenial lesion (MERS). BMJ Case Rep 2013: doi:10.1136/bcr-2013008644 .

9. Fuchigami T, Goto K, Hasegawa M, et al. A 4-yearold girl with clinically mild encephalopathy with a reversible splenial lesion associated with rotavirus infection. J Infect Chemother 2013; 19: 149-153.

10. Mazur-Melewska K, Jonczyk-Potoczna, Szpura K, et al. Transient lesion in the splenium of the corpus callosum due to rotavirus infection. Childs Nerv Syst 2015; 31: 997-1000.

11. Fukuda S, Kishi K, Yasuda K, Sejima H, Yamaguchi S. Rotavirus-associated encephalopathy with a reversible splenial lesion. Pediatr Neurol 2009; 40: 131-133.

12. Kato Z, Orii KE, Morimoto M, et al. A transient lesion in the corpus callosum during rotavirus infection. Pediatr Neurol 2009; 41: 467-469.

13. Arawaka C, Fujita Y, Imai Y, et al. Detection of group A rotavirus RNA and antigens in serum and cerebrospinal fluid from two children with clinically mild encephalopathy with a reversible splenial lesion. Jpn J Infect Dis 2011; 64: 204-207.

14. Matsuoka T, Yodoshi T, Sugai M, et al. A case of mild encephalopathy with a reversible splenial lesion associated with G5P[6]rotavirus infection. Case Rep Pediatr 2013: doi: 10.1155/2013/197163.

15. Feraco P, Porretti G, Marchiò G, Bellizzi M, Recla M. Mild encephalitis/encephalopathy with reversible splenial lesion (MERS) due to cytomegalovirus: case report and review of the literature. Neuropediatrics 2018; 49: 68-71.

16. Ka A, Britton $\mathrm{P}$, Troedson $\mathrm{C}$, et al. Mild encephalopathy with reversible splenial lesion: an important differential of encephalitis. Eur J Paediatr Neurol 2015; 19: 377-382. 
17. Fang Q, Chen L, Chen Q, Lin Z, Yang F. Clinically mild encephalitis/encephalopathy with a reversible splenial lesion of corpus callosum in Chinese children. Brain Dev 2017; 39: 321-326.

18. Takanashi JI, Barkovich AJ, Yamaguchi KI, Kohno Y. Influenza-associated encephalitis/encephalopathy with a reversible lesion in the splenium of the corpus callosum: a case report and literature. AJNR Am J Neuroradiol 2004; 25: 798-802.

19. Britton PN, Dale RC, Blyth CC, et al; ACE study investigators and PAEDS network Influenzaassociated encephalitis/encephalopathy identified by the Australian childhood encephalitis study 20132015. Pediatr Infect Dis J 2017; 36: 1021-1026.

20. Vanderschueren G, Schotsmans K, Maréchal E, Crols R. Mild encephalitis with reversible splenial (MERS) lesion syndrome due to Influenza B virus. Pract Neurol 2018; 18: 391-392.

21. Fluss J, Ferey S, Menache-Starobinski C, Delavelle J, Van Bogaert P, Vargas MI. Mild influenza-associated encephalopathy/encephalitis with a reversible splenial lesion in a Caucasian child with additional cerebellar features. Eur J Paediatr Neurol 2010; 14: 97-100.

22. Ganapathy S, Ey EH, Wolfson BJ, Khan N. Transient isolated lesion of the splenium associated with clinically mild influenza encephalitis. Pediatr Radiol 2008; 38: 1243-1245.

23. Matsubara K, Kodera M, Nigami H, Yura K, Fukaya T. Reversible splenial lesion in influenza virus encephalopathy Pediatr Neurol 2007; 37: 431-434.

24. Morichi S, Kawashima H, Ioi H, Yamanaka G, Kashiwagi Y, Hoshika A. High production of interleukin-10 and interferon- $\gamma$ in influenzaassociated MERS in the early phase. Pediatr Int 2012; 54: 536-562.

25. Shah S, Keil A, Gara K, Gara K, Nagarajan L. Neurologic complication of influenza. J Child Neurol 2014; 29: NP49-NP53.

26. Iwata A, Matsubara K, Nigami H, Kamimura K, Fukaya T. Reversible splenial lesion associated with Novel Influenza A (H1N1) viral infection. Pediatr Neurol 2010; 42: 447-450.

27. Miyata R, Tanuma N, Hayashi M, et al. Oxidative stress in patients with clinically mild encephalitis/ encephalopathy with a reversible splenial lesion (MERS). Brain Dev 2012; 34: 124-127.

28. Chen WX, Liu HS, Yang SD, et al. Reversible splenial lesion syndrome in children: retrospective study and summary of case series. Brain Dev 2016; 38: 915-927.
29. Halpern LA, Agyeman P, Steinlin M, El-Koussy M, Grunt S. Mild encephalopathy with splenial lesion and parainfluenza virus infection. Pediatr Neurol 2013; 48: 252-254.

30. Hatanaka M, Kashiwagi M, Tanabe T, Nakahara $\mathrm{H}$, Ohta K, Tamai H. Overlapping MERS and mild AESD caused by HHV-6 infection. Brain Dev 2015; 37: 334-338.

31. Hara M, Mizuochi T, Kawano G, et al. A case of clinically mild encephalitis with a reversible splenial lesion (MERS) after mumps vaccination. Brain Dev 2011; 33: 842-844.

32. Takanashi JI, Shiihara T, Hasegawa T, et al. Clinically mild encephalitis with a reversible splenial lesion (MERS) after mumps vaccination. J Neurol Sci 2015; 349: 226-228.

33. Suzuki H, Kusaka T, Okada H. Clinically mild encephalitis/encephalopathy with a reversible splenial lesion caused by human parvovirus B19 infection: a case of two brothers with hereditary spherocytosis. Pediatr Neurol 2014; 51: 470-472.

34. Yagamguchi H, Ishida T, Yokoi T, et al. Clinically mild encephalitis/encephalopathy with a reversible splenial lesion accompanied by Epstein-Barr virus hemophagocytic lymphohistiocytosis: a case report and review of the literature. J Pediatr Hematol Oncol 2017; 39: e92-e96.

35. Azuma J, Nabatame S, Katsura T, et al. Marked elevation of urinary $\beta 2$-microglobulin in patients with reversible splenial lesions: a small case series. J Neurol Sci 2016; 368: 109-112.

36. Ueda N, Minami S, Akimoto M. Mycoplasma pneumoniae-associated mild encephalitis/ encephalopathy with a reversible splenial lesion: report of two pediatric cases and a comprehensive literature review. BMC Infect Dis 2016; 16: 671.

37. Yuan ZF, Shen J, Mao SS, et al. Clinically mild encephalitis/encephalopathy with a reversible splenial lesion associated with Mycoplasma pneumoniae infection. BMC Infect Dis 2016; 16: 230.

38. Kawagoshi R, Ono J. A case of Mycoplasmaassociated encephalitis/encephalopathy with a reversible splenial lesion. Meiwa Igaku Zasshi 2015; 2: 35-40. [in Japanese].

39. Tokunaga Y. 1 girl case who showed transient of corpus callosum lesion with P-248 mycoplasmal diseases. No to Hattatsu 2008; 40(Suppl): 396. [in Japanese].

40. Ohgoshi Y, Sakai T, Nonaka S, Nakamura Y, Hosaki A, Bessho F. A case of mild encephalitis/ encephalopathy with a reversible splenial lesion associated with Mycoplasma pneumonia. Nihon Shonika Gakkai Zasshi 2009; 113: 350. [in Japanese]. 
41. Nakamoto T, Tanaka K, Koga H, Kan N, Takahashi S. A case of mild encephalitis/encephalopathy with a reversible splenial lesion during Mycoplasma pneumoniae infection. Nihon Shonika Gakkasi Zasshi 2012; 116: 1255. [in Japanese].

42. UchidaY,MoritaH,MiyazakiK,AdachiS, Tatebayashi $\mathrm{K}$, Kaneko H. A case of menimgoencephalitis with a reversible splenial lesion due to Mycoplasma pneumoniae infection. Shoni Kansen Menneki 2013; 25: 201-202. [in Japanese].

43. Osuka S, Imai H, Ishikawa E, et al. Mild encephalitis/ encephalopathy with a reversible splenial lesion: evaluation by diffusion tensor imaging. Two case reports. Neorol Med Chir (Tokyo) 2010; 50: 11181122.

44. Notebaert A, Willems J, Coucke L, Van Coster R, Verhelst $\mathrm{H}$. Expanding the spectrum of MERS type 2 lesions, a particular form of encephalitis. Pediatr Neurol 2013; 48: 135-138.

45. Jain S, Patel B, Bhatt GC. Enteroviral encephalitis in children: clinical features, pathophysiology and treatment advances. Pathog Glob Health 2014; 108: 216-222.

46. Pallansch MA, Roos R. Enteroviruses: polioviruses, coxsackieviruses, echoviruses, and newer enteroviruses. . In: Knipe DM, Howley PM (eds). Fields Virology (5th edt).Philadelphia: Lippincott Williams \& Wilkins, 2007: 839-894.

47. Hoshino A, Saitoh M, Oka A, et al. Epidemiology of acute encephalopathy in Japan, with emphasis on the association of viruses and syndromes. Brain Dev 2012; 34:337-343.

48. Kurokawa Y, Masuda H, Kobayashi H, et al. Effective therapy with infliximab for clinically mild encephalitis/encephalopathy with a reversible splenial lesion in an infant with Kawasaki disease. Nihon Rinsho Meneki Gakkai Kaishi 2017; 40: 190195.

49. Benschop KS, Geeraedts F, Beuvink B, et al; VIROTypeNed Increase in ECHOvirus 6 infections associated with neurological symptoms in the Netherlands, June to August 2016. Euro Surveil 2016; 21: 30351.

50. Hosoda A, Gatayama R, Moriyama S, et al. The first case of recurrent ultra late onset group $B$ streptococcal sepsis in a 3-year-old child. IDCases 2017; 7: 16-18.
51. Hashimoto Y, Takanashi JI, Kaiho K, et al. A splenial lesion with transiently reduced diffusion in clinically mild encephalitis is not always reversible. A case report. Brain Dev 2009; 31 :710-712.

52. Ikeno M, Abe S, Kurahashi H, Takasu M, Shimizu T, Okumura A. Gastric perforation and critical illness polyneuropathy after steroid treatment in a patient with encephalitis/encephalopathy with transient splenial lesion. Brain Dev 2016; 39: 356-360.

53. Nozaki F, Kumada T, Miyajima T, et al. Reversible splenic lesion in a patient with febrile infection related epilepsy syndrome. Neuropediatrics 2013; 44: 291-294.

54. Pan JJ, Zhao YY, Lu C, Hu YH, Yang Y. Mild encephalitis/encephalopathy with a reversible splenial lesion: five cases and a literature review. Neurol Sci 2015; 36: 2043-2051.

55. Sun D, Chen WH, BaralcS, et al. Mild encephalopathy/ encephalitis with a reversible splenial lesion (MERS): a report of five neonatal cases. J Huazhong Univ Sci Technol [Med Sci] 2017; 37: 433-438.

56. Itamura S, Kamada M, Nakagawa N. Kawasaki disease complicated with reversible splenial lesion and acute myocarditis. Pediatr Cardiol 2011; 32: 696699.

57. Takanashi JI, Shirai K, Sugawara Y, Okamoto Y, Obonai T, Terada H. Kawasaki disease complicated by mild encephalopathy with a reversible splenial lesion (MERS). J Neurol Sci 2012; 315: 167-169.

58. Kometani H, Kawatani M, Ohta G, et al. Marked elevation of interleukin- 6 in mild encephalopathy with a reversible splenial lesion (MERS) associated with acute focal bacterial nephritis caused by Enterococcus faecalis. Brain Dev 2013; 36: 551-553.

59. Imamura T, Takanashi JI, Yasugi J, Terada H, Nishimura A. Sisters with clinically mild encephalopathy with a reversible splenial lesion (MERS)-like features; familial MERS? J Neurol Sci 2010; 290: 153-156.

60. Yıldız AE, Maraş Genç H, Gürkaș E, Akmaz Ünlü H, Öncel İH, Güven A. Mild encephalitis/ encephalopathy with a reversible splenial lesion in children. Diagn Interv Radiol 2018; 24: 108-112. 